\title{
Development of accurate well models for numerical reservoir simulation
}

\author{
Shengsheng Zhang, Zhifeng Liu ${ }^{\circledR}$, Anfeng Shi, Xiaohong Wang \\ Department of Thermal Science and Energy Engineering, University of Science and Technology of China, Hefei 230026, P. R. China
}

(Received April 10, 2019; revised May 11, 2019; accepted June 2, 2019; available online June 6, 2019)

Citation:

Zhang, S., Liu, Z., Shi, A., Wang, X.

Development of accurate well models for numerical reservoir simulation. Advances in Geo-Energy Research, 2019, 3(3): 250-257, doi: 10.26804/ager.2019.03.03.

Corresponding author:

*E-mail: lzf13@ustc.edu.cn

Keywords:

Peaceman well model

equivalent radius

bottom-hole pressure

source term compensation method

pattern competition method

\begin{abstract}
:
The Peaceman well model has been an industrial standard in numerical reservoir simulation. With the help of defined equivalent radius, the bottom-hole inflow or outflow flux is calculated as being proportional to the difference between the bottom-hole pressure and the well-grid block pressure. We show in this paper that, although the bottom-hole flux is calculated accurately in the Peaceman well model, some significant errors of pressure arise near the well for a large value of the length-to-width ratio of the mesh. We propose two alternative methods, the source term compensation method and the pattern competition method, both of which are based on an analytic solution induced by the source term for the homogeneous case. In the source term compensation method, the auxiliary pressure, which strictly satisfies the Laplace equation, is defined and solved instead of the original pressure variable only satisfying the Poisson equation. In the pattern competition method, different flow patterns including the linear flow pattern and radial flow pattern are considered. Each flow pattern corresponds to an specific value of the transmissibility of the two adjacent grid blocks. The smallest transmissibility will outcompete, and be used for solving the discrete pressure equations. Numerical results show that not only the bottom-hole flux but also the pressure fields can be calculated accurately using both of proposed methods.
\end{abstract}

\section{Introduction}

Numerical reservoir simulation for oil and gas production must account for the presence of wells. It is necessary to use grid blocks whose horizontal dimensions are much larger than the diameter of a well. As a result of the traditional numerical algorithm, the pressure calculated for a well block is greatly different from the flowing bottom-hole pressure (BHP) of the well. Well models are needed to relate these two different pressures for the grid blocks where the wells are located (Williamson, 1981).

Peaceman's well model is usually the default one in a general reservoir simulator. His model gave a proper interpretation of a well-block pressure, and indicated how it relates to the flowing BHP by introducing the concept of equivalent radius. The initial Peaceman's finite difference well model was for the well located in the center of a square grid block for single phase flow (Peaceman, 1978). It has been extended in various directions, including rectangular gridblocks, anisotropic reservoirs, horizontal wells, wells located anywhere in the grids, and multiphase flow, as well as incorporating the gravity force, skin, and non-Darcy effects (Peaceman, 1983; Peaceman, 1990; Peaceman, 1993; Palagi et al., 1994; Peaceman, 1994; Mochizuki et al., 1995; Peaceman, 1995; Ding, 1998;
Peaceman, 2003; Chen et al., 2009; Dumkwu et al., 2012; Aavatsmark, 2016).

Some scholars pointed out that errors using standard (default) well indices are significant in many cases, especially for the example involving a complex (herringbone) multilateral well (Wolfsteiner et al., 2003). Durlofsky presented an analytical model to approximate the productivity of vertical wells in terms of an effective permeability coupled with a nearwell skin (Durlofsky, 2000). Ding and Jeannin pointed out that truncation errors of the standard approach might be large in the near well region due to the well singularity, and they proposed a multi-point flux approximation to improve the accuracy for the well flux calculation (Ding et al., 2007). Ding introduced the layer potential for representing the 3D pressure distribution in the vicinity of wells (Ding, 1996), and proposed the nearwell upscaling technique to improve the coarse-grid simulation accuracy for well productivities (Ding, 2004). Similar local grid refinements (LGR) methods are also employed to model laminated reservoirs (Correia et al., 2018) and fractured porous media (Fumagalli, 2010). Well models with high precision is very helpful for building a simple model summarizing the interwell connectivities and the associated drainage area of each well before processing expensive and detailed multiphase reservoir simulations (Noetinger, 2015; Mirzayev et al., 2019). 
In this paper, we will show that when the length-to-width ratio of the mesh is large, even the single phase Peaceman well model will cause some significant pressure errors near the well. To improve the accuracy, two methods that can take place of the Peaceman well model are proposed in the case of homogeneous medium. The accuracy of the proposed methods is indicated through numerical results.

The paper is organized as follows. In section 2, the original Peaceman well model is reviewed. In section 3 and 4, the source term compensation method and pattern competition methods are introduced respectively. Numerical test is provided in section 5. Takeaways and potential extensions of the proposed methods are discussed in section 6 .

\section{Brief review of peaceman well model}

When modeling reservoir behavior by numerical methods, inevitably the horizontal dimensions of any grid block containing a well are much larger than the wellbore radius (See Fig. 1). The traditional numerical algorithm provides the node equation in the well grid for single steady flow:

$$
\begin{aligned}
q & =\frac{\rho k}{\mu}\left[\frac{\Delta y}{\Delta x}\left(p^{(i-1, j)}+p^{(i+1, j)}\right)+\frac{\Delta x}{\Delta y}\left(p^{(i, j-1)}+p^{(i, j+1)}\right)\right] \\
& -\frac{2 \rho k}{\mu}\left(\frac{\Delta x}{\Delta y}+\frac{\Delta y}{\Delta x}\right) p^{(i, j)}
\end{aligned}
$$

where $\rho$ is the density, $k$ is the permeability, $\mu$ is the viscosity, and $\Delta x, \Delta y$ are the space steps in $x$ and $y$ directions, respectively. $q$ is the bottom-hole flow rate (The positive values of $q$ represent flow into the well, and negative values represent flow out of the well). Obviously, the calculated well block pressure $p^{(i, j)}$ will be greatly different from the bottom hole pressure $p_{w}$ if using the node Eq. (1). A numerical well model has to be constructed to relate the bottom-hole flow rate $q$ to the difference of the well block pressure and the bottom hole pressure $p^{(i, j)}-p_{w}$. Then the concept of equivalent radius $r_{e}$ is introduced.

An equivalent radius $r_{e}$ is defined for the well block, at which the steady-state flowing pressure for the actual well is equal to the numerically calculated pressure $p^{(i, j)}$. For the incompressible steady radial flow described by the Laplace equation $\frac{d}{d r}\left(r \frac{d p}{d r}\right)=0$, the definition of $r_{e}$ gives:

$$
q=\frac{2 \pi \rho k}{\mu} \frac{\left[p^{(i, j)}-p_{w}\right]}{\ln \left(r_{e} / r_{w}\right)}
$$

In the reference (Peaceman, 1983), Peaceman indicated that for the well located in the center the rectangular well block, the equivalent radius $r_{e}$ can be calculated as:

$$
r_{e}=\frac{e^{-\gamma}}{4}\left(\Delta x^{2}+\Delta y^{2}\right)^{1 / 2}
$$

with $\gamma=0.5772$ being the Euler's constant. When $\Delta x=\Delta y$, Eq. (3) provides $r_{e} \approx 0.2079 \Delta x$.

Eqs. (1)-(3) are just the Peaceman well model, which is the standard and default one in the numerical reservoir simula-

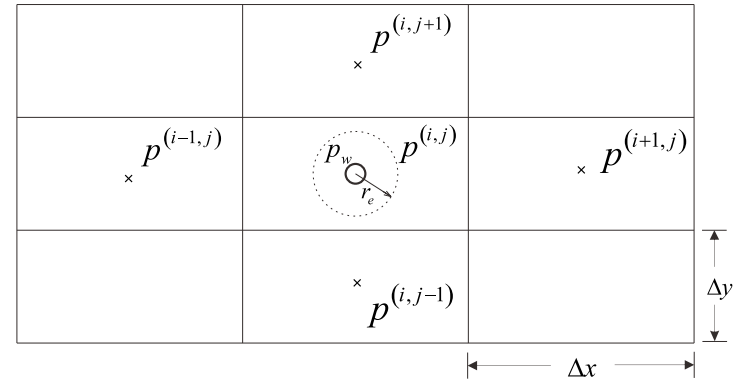

Fig. 1. Block $(i, j)$ containing a well and its four neighboring blocks.

tion. The reason why it is adopted widely is that it predicts the bottom-hole flux rate $q$ quite accurately.

Abou-Kassem and Aziz defined another equivalent radius $r_{e, \text { ana }}$ through analytic analysis (Abou-Kassem et al., 1985), which is different from the $r_{e}$ defined above. The "analytic equivalent radius $r_{e, \text { ana }}$ " can be obtained as described below. According to the analytical solution induced by the source term $p=\frac{\mu q}{2 \pi \rho k} \ln r$, the discrete pressures around the well block can be expressed as:

$$
\begin{aligned}
& p^{(i-1, j)}=p^{(i+1, j)}=\frac{\mu q}{2 \pi \rho k} \ln \Delta x, \\
& p^{(i, j-1)}=p^{(i, j+1)}=\frac{\mu q}{2 \pi \rho k} \ln \Delta y
\end{aligned}
$$

Substituting Eq. (4) into Eq. (1), we can obtain that:

$$
p^{(i, j)}=\frac{\frac{q \mu}{2 \rho k}\left(\frac{\Delta y}{\pi \Delta x} \ln \Delta x+\frac{\Delta x}{\pi \Delta y} \ln \Delta y-1\right)}{\left(\frac{\Delta x}{\Delta y}+\frac{\Delta y}{\Delta x}\right)}
$$

According to the definition of $r_{e, \text { ana }}$ which implies $p^{(i, j)}=$ $\frac{\mu q}{2 \pi \rho k} \ln r_{e, \text { ana }}$, the value of $r_{e, \text { ana }}$ can be calculated as:

$$
\ln r_{e, \text { ana }}=\frac{\left(\frac{\Delta y}{\Delta x} \ln \Delta x+\frac{\Delta x}{\Delta y} \ln \Delta y-\pi\right)}{\left(\frac{\Delta x}{\Delta y}+\frac{\Delta y}{\Delta x}\right)}
$$

When $\Delta x=\Delta y$, Eq. (6) provides $r_{e, a n a} \approx 0.1985 \Delta x$, which is very close to the equivalent radius $r_{e} \approx 0.2079 \Delta x$ defined by Peaceman. However if $\Delta x \neq \Delta y$, the difference between $r_{e}$ and $r_{e, a n a}$ is significant, especially for the large ratio of $\Delta x / \Delta y$.

We performed a simple test of the Peaceman well model here. A well is located in the center of a circular region (see Fig. 2a). In this article, we use lower-case letters to represent the original variables, and the capital ones to represent their corresponding dimensionless quantities. According to this convention, the dimensionless radius of the region and the well in this test is set as $R_{b}=1$ and $R_{w}=10^{-5}$, respectively. The dimensionless pressures at the outer boundary and at the bottomhole are also given: $\left.P\right|_{R=R_{b}}=0,\left.P\right|_{R=R_{w}}=-\frac{1}{2 \pi} \ln \left(10^{-5}\right)$. Actually, this boundary condition just corresponds to the exact solution of the dimensionless Poisson equation $\nabla^{2} P=Q \delta(\mathbf{R})$ with its dimensionless source term strength $Q=-1$. That is, $P=\frac{Q}{2 \pi} \ln R$. This analytic solution will be used to check the accuracy of Peaceman well model. 


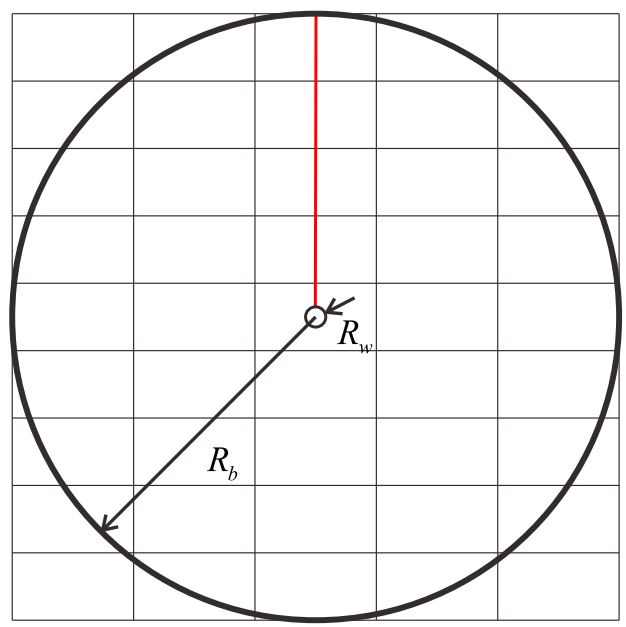

(a)

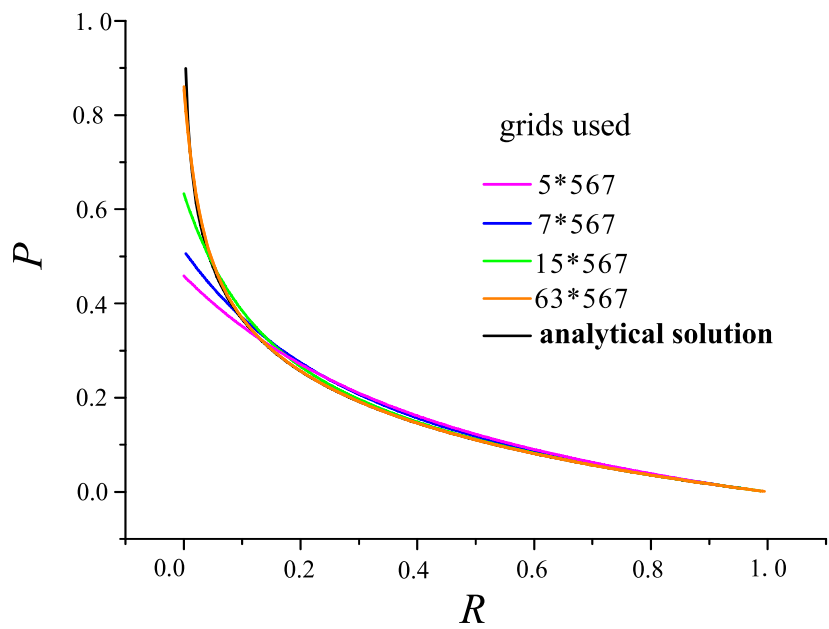

(b)

Fig. 2. Radial pressure distribution along the red line (shown in (a)) calculated from the Peaceman well model.

Table 1. The calculated dimensionless source strength $Q$ under different grids (The exact value is $Q=-1$.).

\begin{tabular}{lll}
\hline Number of the grids & $\begin{array}{l}\text { The calculated value of } Q \text { when using } \\
R_{e} \text { defined in Eq. (3) }\end{array}$ & $\begin{array}{l}\text { The calculated value of } Q \text { when using } \\
R_{e, \text { ana }} \text { defined in Eq. (6) }\end{array}$ \\
\hline $5 \times 5$ & -1.0009 & -0.9968 \\
$7 \times 7$ & -1.0000 & -0.9960 \\
$15 \times 15$ & -1.0000 & -0.9960 \\
$5 \times 567$ & -0.9998 & -1.3289 \\
$7 \times 567$ & -1.0000 & -1.2778 \\
$15 \times 567$ & -1.0000 & -1.1805 \\
$21 \times 567$ & -1.0000 & -1.1442 \\
$63 \times 567$ & -1.0000 & -1.0510 \\
\hline
\end{tabular}

In Table 1, the calculated dimensionless source strength $Q$ is shown. It shows that Peaceman well model predicts the source strength $Q$ accurately. However, if using the analytic equivalent radius $R_{e, \text { ana }}$ defined in Eq. (6), large errors would be caused under the grids where $\Delta X \neq \Delta Y$. As mentioned above, this is reason why Peaceman well model is widely adopted.

In Fig. 2, the calculated dimensionless pressure distributions along the radial direction are shown for different grids when using Peaceman well model. It can be seen that, although the source strength $Q$ is calculated accurately, there are still some significant errors of the calculated pressure $P$ at the grid blocks near the well when the length-to-width ratio of the mesh is large. In the next sections, we will propose two new algorithms to improve the calculation.

\section{Source term compensation method}

Let's recall Eq. (1) for the well block. When using Eq. (1), it is implied that the pressure is distributed linearly in the well block and its neighbors. Of course, it is not the case when source term exists. For homogeneous case, since the analytic Green's function of the source term is known, one can intro-

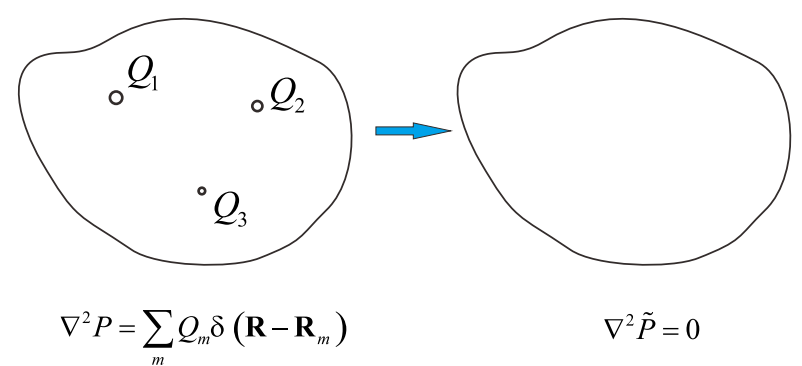

Fig. 3. Sketch map of transforming the Poisson equation into the Laplace one by defining the the auxiliary pressure $\tilde{P}$.

duce a compensatory term to obtain a new linearly distributed pressure field. Then the new pressure field can be solved accurately as usual using the traditional 5-point stencil. The details are described as follow.

Let's consider a 2D domain with several source terms, as shown in Fig. 3. Due to the existence of the source terms, the pressure distribution $P(\mathbf{R})$ is far from linear, especially at the area near the sources. Since the pressure induced by the source term $Q_{m}$ can be expressed analytically as $P_{m}=\frac{Q_{m}}{2 \pi} \ln R_{m}+C_{m}$ (the constant $C_{m}$ can be omitted), one can introduce an 
auxiliary pressure $\tilde{P}$ :

$$
\tilde{P}=P-\sum_{m} P_{m}=P-\sum_{m} \frac{Q_{m}}{2 \pi} \ln R_{m}
$$

where $R_{m}$ is the distance from the observed point to the source $Q_{m}$. Thus, the auxiliary pressure strictly satisfies the Laplace equation $\nabla^{2} \tilde{P}=0$ in the domain. The original pressure variable $P$ actually satisfies the Poisson equation $\nabla^{2} P=$ $\sum_{m} Q_{m} \delta\left(\mathbf{R}-\mathbf{R}_{m}\right)$. The traditional 5-point stencil works well for solving the auxiliary pressure:

$$
\begin{aligned}
& \frac{\Delta Y}{\Delta X}\left(\tilde{P}^{(i-1, j)}+\tilde{P}^{(i+1, j)}\right)+\frac{\Delta X}{\Delta Y}\left(\tilde{P}^{(i, j-1)}+\tilde{P}^{(i, j+1)}\right) \\
& -2\left(\frac{\Delta X}{\Delta Y}+\frac{\Delta Y}{\Delta X}\right) \tilde{P}^{(i, j)}=0
\end{aligned}
$$

If all the source strength $Q_{m}$ are known, one can solve Eq. (8) directly. Notice that in this situation, the boundary conditions of the auxiliary pressure $\tilde{P}$ can be obtained directly according to Eq. (7). After getting the auxiliary pressure $\tilde{P}$, the original pressure field $P$ can be calculated also according to Eq. (7).

If not all the source strength $Q_{m}$ are known, it means that the boundary conditions of the auxiliary pressure $\tilde{P}$ can not be obtained directly from Eq. (7). Suppose $Q_{k}$ is unknown and located in the cell $\left(i_{k}, j_{k}\right)$; instead, the bottom hole pressure and the well radius $\left(P_{w, k}, R_{w, k}\right)$ are known. Eq. (8) only provides the same number of equations as the number of nodes. One additional equation is needed since an extra unknown variable $Q_{m}$ is involved. This additional equation is just the fixed bottom hole pressure condition of $i$ th well, which is

$$
\tilde{P}^{\left(i_{k}, j_{k}\right)}=P_{w, k}-\sum_{m \neq k} \frac{Q_{m}}{2 \pi} \ln R_{m}^{\left(i_{k}, j_{k}\right)}-\frac{Q_{k}}{2 \pi} \ln R_{w, k}
$$

where $R_{m}^{\left(i_{k}, j_{k}\right)}$ is the distance from the center of the grid $\left(i_{k}, j_{k}\right)$ to the location of the source $Q_{m}$.

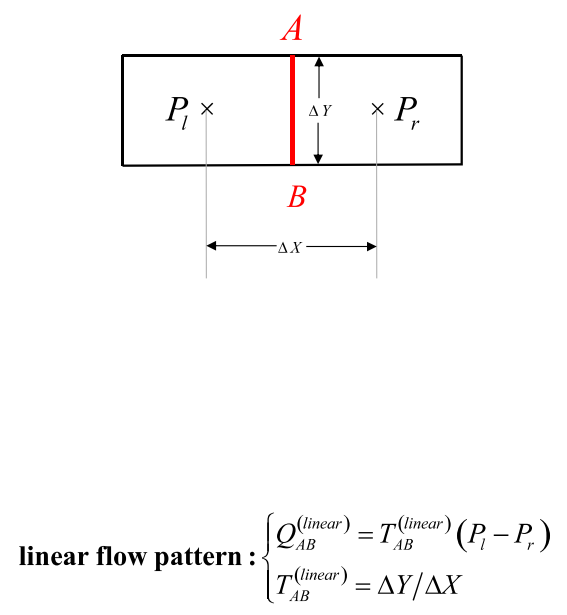

(a)
For each unknown $Q_{k}$, a supplementary equation similar as Eq. (9) can be obtained. Thus the total number of the unknown variables including the set of discrete auxiliary pressure $\left\{\tilde{P}^{(i, j)}\right\}$ and the set of unknown source terms $\left\{Q_{k}\right\}$ matches the number of equations, then $\left\{\tilde{P}^{(i, j)}\right\}$ and $\left\{Q_{k}\right\}$ can be solved out.

If performing this source term compensation method to the problem described in Fig. 2a, the numerical results will reproduce the analytic one. This is because the boundary condition of the auxiliary pressure is just $\left.\tilde{P}\right|_{r_{b}}=0$, which leads the solution $\tilde{P}=0$ of the Laplace equation $\nabla^{2} \tilde{P}=0$ even in the discrete form. Another numerical test is performed and the numerical results are compared to the Peaceman model in the section 5 .

\section{Pattern competition method}

When solving the Laplace or Poisson equations numerically, it is essential to determine the transmissibility of the adjacent two grids. For example, in the traditional 5-point stencil (see Fig. 4a), the dimensionless flux rate across the interface $\overline{A B}$ can be approximated as $Q_{A B}=\frac{\Delta Y}{\Delta X}\left(P_{l}-P_{r}\right)$; that is, the transmissibility $T_{A B}=\frac{\Delta Y}{\Delta X}$. Actually, this value of the transmissibility is calculated under the linear flow pattern (See Fig. 4a). Artus et al. pointed out that in the case of transient flowing for the very low permeability of the formation, the linear transmissibility derivations overestimate the production forecast, and proposed a new approach for transmissibility derivations based on numerical integrations of source point solutions (Arus et al., 2012). We rewrite the transmissibility for linear flow pattern as:

$$
T_{A B}^{(\text {linear })}=\frac{\Delta Y}{\Delta X}, Q_{A B}^{(\text {linear })}=T_{A B}^{(\text {linear })}\left(P_{l}-P_{r}\right)
$$

If there is a source term $Q$ locating in the domain (see Fig. $4 b$ ), the transmissibility can be calculated as following. The

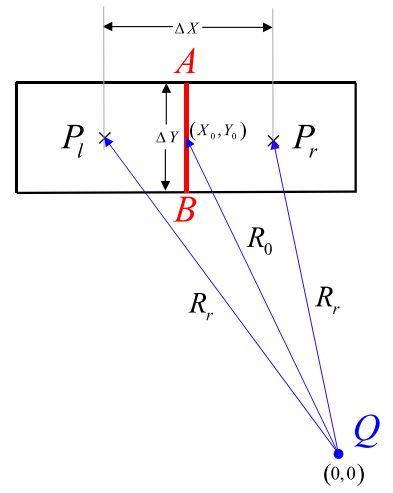

radial flow pattern : $\left\{\begin{array}{l}Q_{A B}^{(\text {radial })}=T_{A B}^{(\text {radial })}\left(P_{l}-P_{r}\right) \\ T_{A B}^{(\text {radial })}=\left.\frac{1}{\ln \left(R_{r} / R_{l}\right)} \operatorname{arctg} \frac{Y}{X_{0}}\right|_{Y_{0}-\Delta Y / 2} ^{X_{0}+\Delta Y / 2}\end{array}\right.$

(b)

Fig. 4. Sketch map of calculating the transmissibility for linear flow pattern (a) and radial flow pattern (b). 
source term $Q$ corresponds to the analytic solution $P=\frac{Q}{2 \pi} \ln R$, thus

$$
\left\{\begin{array}{l}
P_{r}=\frac{Q}{2 \pi} \ln R_{r} \\
P_{l}=\frac{Q}{2 \pi} \ln R_{l}
\end{array}\right.
$$

Then,

$$
Q=\frac{2 \pi}{\ln R_{r} / R_{l}}\left(P_{r}-P_{l}\right)
$$

The flux flow rate across the interface $\overline{A B}$ can be calculated according to the analytic solution $P=\frac{Q}{2 \pi} \ln R$.

$Q_{A B}^{(\text {radial })}=-\left.\int_{Y_{0}-\frac{\Delta Y}{2}}^{Y_{0}+\frac{\Delta Y}{2}} \frac{\partial P}{\partial X}\right|_{X=X_{0}} d Y=-\frac{Q}{2 \pi} \int_{Y_{0}-\frac{\Delta Y}{2}}^{Y_{0}+\frac{\Delta Y}{2}} \frac{X_{0}}{X_{0}^{2}+Y^{2}} d Y$

$$
=-\left.\frac{Q}{2 \pi} \operatorname{arctg} \frac{Y}{X_{0}}\right|_{Y_{0}-\frac{\Delta Y}{2}} ^{Y_{0}+\frac{\Delta Y}{2}}
$$

Combing Eq. (12) and Eq. (13), one can obtain the transmissibility under radial flow pattern:

$$
\begin{aligned}
Q_{A B}^{(\text {radial })} & =T_{A B}^{(\text {radial })}\left(P_{l}-P_{r}\right), \\
T_{A B}^{(\text {radial })} & =\left.\frac{1}{\ln \left(R_{r} / R_{l}\right)} \operatorname{arctg} \frac{Y}{X_{0}}\right|_{Y_{0}-\frac{\Delta Y}{2}} ^{Y_{0}+\frac{\Delta Y}{2}}
\end{aligned}
$$

It is necessary to point out that when $\Delta X / R_{0} \rightarrow 0$ and $\Delta Y / R_{0} \rightarrow 0, T_{A B}^{(\text {radial })}=T_{A B}^{(\text {linear })}$.

Proof. According to the L'Hospitals Rule, for a given $R_{0}$, when $\Delta X \rightarrow 0$ and $\Delta Y \rightarrow 0$, we have:

$$
\begin{aligned}
& \lim _{\substack{\Delta X \rightarrow 0 \\
\Delta Y \rightarrow 0}} T_{A B}^{(\text {radial })}=\left.\lim _{\substack{\Delta X \rightarrow 0 \\
\Delta Y \rightarrow 0}} \frac{1}{\ln \left(R_{r} / R_{l}\right)} \operatorname{arctg} \frac{Y}{X_{0}}\right|_{Y_{0}-\frac{\Delta Y}{2}} ^{Y_{0}+\frac{\Delta Y}{2}} \\
& =\left.\lim _{\substack{\Delta X \rightarrow 0 \\
\Delta Y \rightarrow 0}} \frac{1}{\ln \frac{\sqrt{\left(X_{0}+\Delta X / 2\right)^{2}+Y_{0}^{2}}}{\sqrt{\left(X_{0}-\Delta X / 2\right)^{2}+Y_{0}^{2}}}} \operatorname{arctg} \frac{Y}{X_{0}}\right|_{Y_{0}-\frac{\Delta Y}{2}} ^{Y_{0}+\frac{\Delta Y}{2}} \\
& =\lim _{\Delta X \rightarrow 0} \frac{\frac{1}{\Delta X}}{\frac{\ln \sqrt{\left(X_{0}+\Delta X / 2\right)^{2}+Y_{0}^{2}}-\ln \sqrt{\left(X_{0}-\Delta X / 2\right)^{2}+Y_{0}^{2}}}{\Delta X}} \lim _{\Delta Y \rightarrow 0} \Delta Y \frac{\left.\operatorname{arctg} \frac{Y}{X_{0}}\right|_{Y_{0}-\frac{\Delta Y}{2}} ^{Y_{0}+\frac{\Delta Y}{2}}}{\Delta Y} \\
& =\lim _{\Delta X \rightarrow 0} \frac{2}{\Delta X\left[\ln \left(X^{2}+Y_{0}^{2}\right)\right]_{X=X_{0}}^{\prime}} \lim _{\Delta Y \rightarrow 0} \Delta Y\left(\operatorname{arctg} \frac{Y}{X_{0}}\right)_{Y=Y_{0}}^{\prime} \\
& =\lim _{\substack{\Delta X \rightarrow 0 \\
\Delta Y \rightarrow 0}} \frac{\Delta Y}{\Delta X} \\
& =\lim _{\substack{\Delta X \rightarrow 0 \\
\Delta Y \rightarrow 0}} T_{A B}^{(\text {linear })}
\end{aligned}
$$

\section{End proof.}

Eq. (15) confirms that when the grid block sizes $\Delta X$ and $\Delta Y$ are small enough, one will get the same numerical results whether using the transmissibility Eq. (10) of the linear flow pattern or Eq. (14) of the radial flow pattern. The problem now is that when the grid size $\Delta X$ and $\Delta Y$ are of limited values, which transmissibility should be used. Another equivalent problem is when several source terms exist, which transmissibility should be used. A conservative choice is the smallest one (Notice that all the transmissibilities are positive). This is why we call this algorithm Pattern Competition Method.

Suppose several source terms exist in the domain. Each transmissibility for the corresponding source is calculated. All the transmissibilities including the one of the linear flow pattern compete with each other, and the smallest one will outcompete. Actually, the transmissibility of the source flow pattern always outcompetes near the corresponding well. At the area far from the wells, all the transmissibilities of the different flow patterns are almost the same according to Eq. (15). This discrete scheme based on the radial analytic solution is like the scheme in the references (Weber, 2004; Ribeiro, 2014), and also can be seemed as a variety of Finite Analytic Method (Liu, 2013).

\section{Numerical test}

The second numerical test is performed in this article. As shown in Fig. 5, there are three wells locates in a rectangle domain. The dimensionless outer boundary pressures, the location of wells, the bottom-hole radii and pressures are also shown in Fig. 5. The three methods, the Peacman well model, the source term compensation method and the pattern competition method are used respectively, and their calculation results are provided in Table 2 and Fig. 6.

The calculated flux rate of each well is given in Table 2 . It is shown that all the three methods can calculate bottom- 


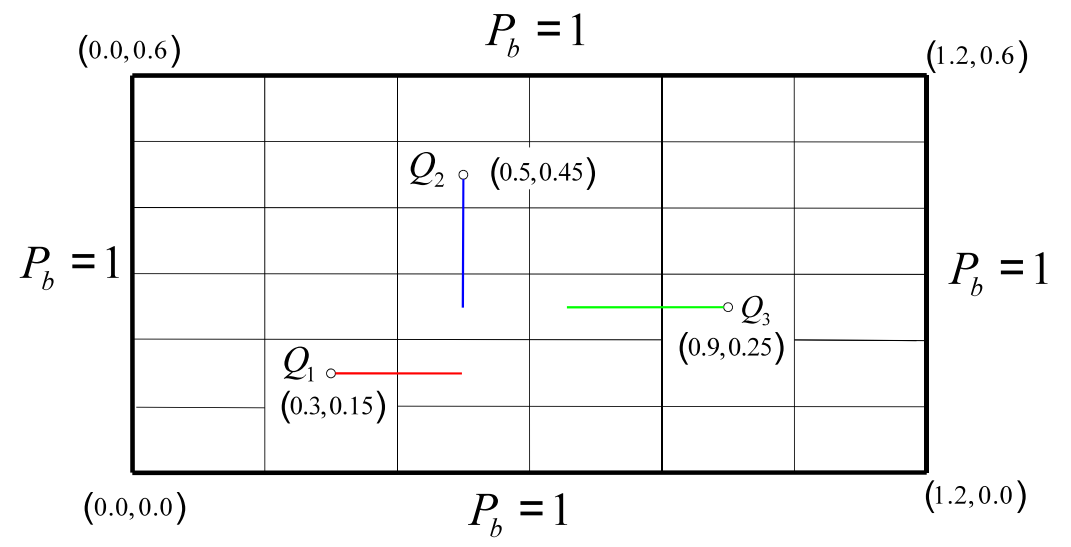

\section{well parameters}

$$
\begin{aligned}
& R_{w 1}=0.5^{*} 10^{-5}, P_{w_{1}}=0.1 \\
& R_{w 2}=1.0^{*} 10^{-5}, P_{w_{2}}=2.0 \\
& R_{w 3}=1.5^{*} 10^{-5}, P_{w_{3}}=0.3
\end{aligned}
$$

Fig. 5. Sketch map of the numerical test example where three source terms locate in the domain.

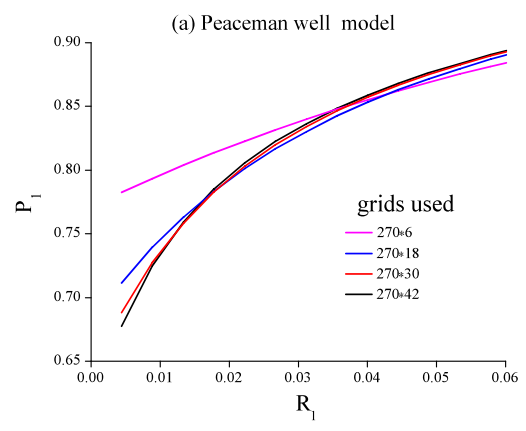

(d) Source term compensation method

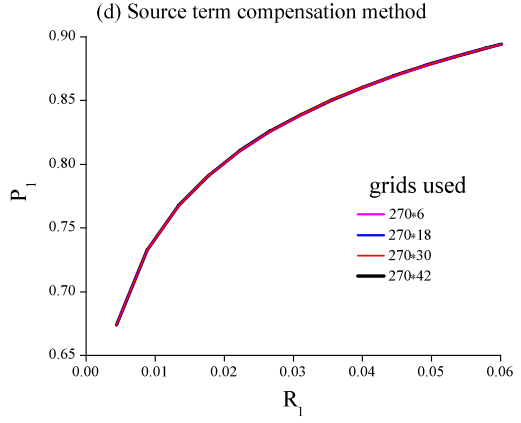

(g) Pattern competition mothod

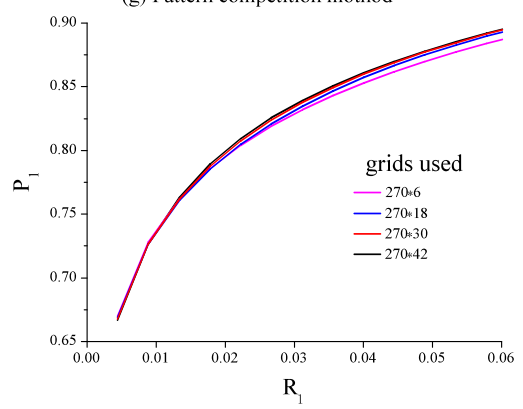

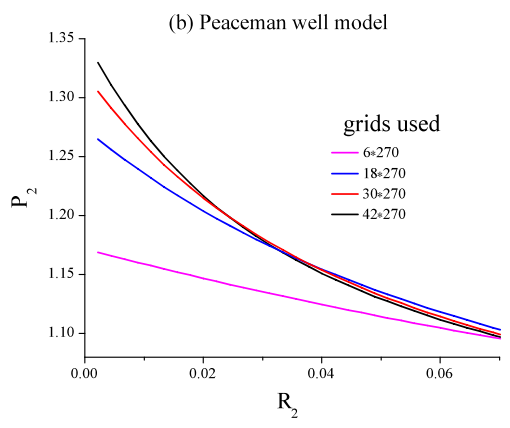

(e) Source term compensation method

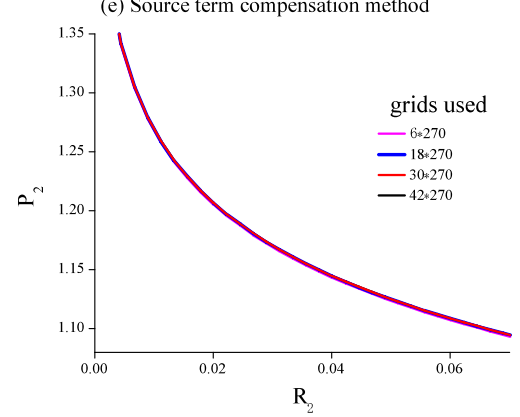

(h) Pattern competition mothod

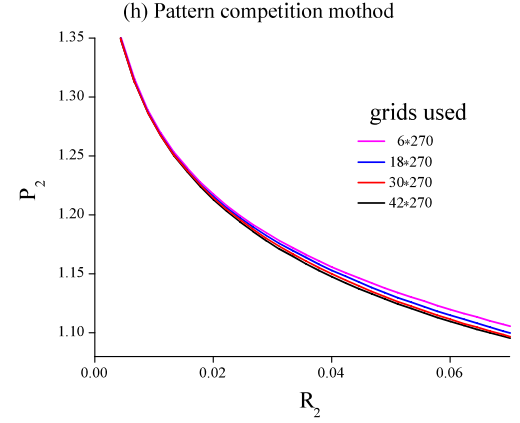

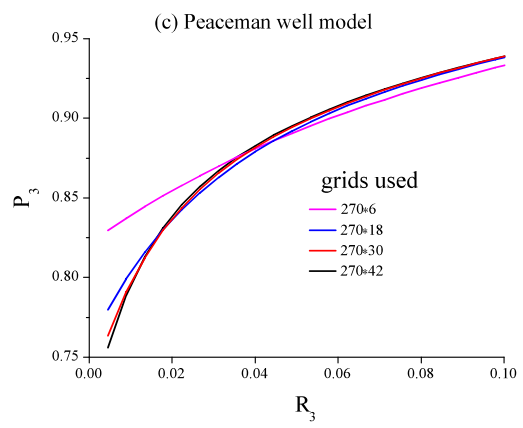

(f) Source term compensation method

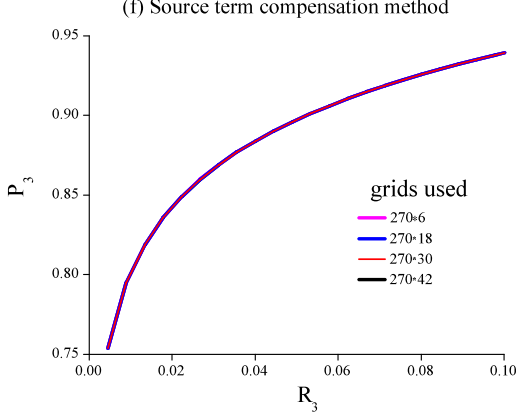

(i) Pattern competition mothod

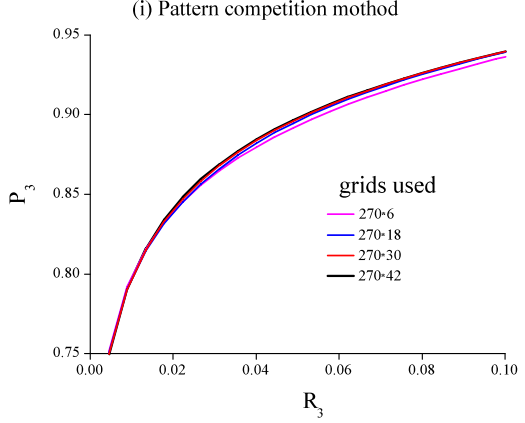

Fig. 6. Radial pressure distribution along the red, blue and green line in Fig. 5. 
Table 2. The comparison of the calculated dimensionless source strength $Q_{1}, Q_{2}, Q_{3}$ under different grids by using the three methods: the Peaceman well model, the source term compensation method and the pattern competition method.

\begin{tabular}{|c|c|c|c|}
\hline Number of the grids & the Peaceman well model & the source term compensation method & the pattern competition method \\
\hline & $Q_{1}=0.53075$ & $Q_{1}=0.53088$ & $Q_{1}=0.52580$ \\
\hline \multirow[t]{3}{*}{$270 \times 6$} & $Q_{2}=-0.56632$ & $Q_{2}=-0.56629$ & $Q_{2}=-0.56117$ \\
\hline & $Q_{3}=0.36998$ & $Q_{3}=0.36992$ & $Q_{3}=0.36998$ \\
\hline & $Q_{1}=0.53106$ & $Q_{1}=0.53108$ & $Q_{1}=0.52623$ \\
\hline \multirow[t]{3}{*}{$270 \times 18$} & $Q_{2}=-0.56656$ & $Q_{2}=-0.56656$ & $Q_{2}=-0.56161$ \\
\hline & $Q_{3}=0.36991$ & $Q_{3}=0.36990$ & $Q_{3}=0.36991$ \\
\hline & $Q_{1}=0.53109$ & $Q_{1}=0.53109$ & $Q_{1}=0.52586$ \\
\hline \multirow[t]{3}{*}{$270 \times 30$} & $Q_{2}=-0.56658$ & $Q_{2}=-0.56658$ & $Q_{2}=-0.56125$ \\
\hline & $Q_{3}=0.36991$ & $Q_{3}=0.36990$ & $Q_{3}=0.36666$ \\
\hline & $Q_{1}=0.53110$ & $Q_{1}=0.53110$ & $Q_{1}=0.52536$ \\
\hline \multirow[t]{3}{*}{$270 \times 42$} & $Q_{2}=-0.56659$ & $Q_{2}=-0.56659$ & $Q_{2}=-0.56074$ \\
\hline & $Q_{3}=0.36990$ & $Q_{3}=0.36990$ & $Q_{3}=0.36635$ \\
\hline & $Q_{1}=0.53366$ & $Q_{1}=0.5311$ & $Q_{1}=0.52632$ \\
\hline \multirow[t]{3}{*}{$6 \times 270$} & $Q_{2}=-0.56897$ & $Q_{2}=-0.56777$ & $Q_{2}=-0.56269$ \\
\hline & $Q_{3}=0.37048$ & $Q_{3}=0.37013$ & $Q_{3}=0.36718$ \\
\hline & $Q_{1}=0.53138$ & $Q_{1}=0.53118$ & $Q_{1}=0.52632$ \\
\hline \multirow[t]{3}{*}{$18 \times 270$} & $Q_{2}=-0.56684$ & $Q_{2}=-0.56669$ & $Q_{2}=-0.56182$ \\
\hline & $Q_{3}=0.36994$ & $Q_{3}=0.36992$ & $Q_{3}=0.36698$ \\
\hline & $Q_{1}=0.53120$ & $Q_{1}=0.53113$ & $Q_{1}=0.52637$ \\
\hline \multirow[t]{3}{*}{$30 \times 270$} & $Q_{2}=-0.56668$ & $Q_{2}=-0.56662$ & $Q_{2}=-0.56180$ \\
\hline & $Q_{3}=0.36992$ & $Q_{3}=0.36991$ & $Q_{3}=0.36700$ \\
\hline & $Q_{1}=0.53115$ & $Q_{1}=0.53112$ & $Q_{1}=0.52635$ \\
\hline \multirow[t]{2}{*}{$42 \times 270$} & $Q_{2}=-0.56663$ & $Q_{2}=-0.56661$ & $Q_{2}=-0.56175$ \\
\hline & $Q_{3}=0.36991$ & $Q_{3}=0.36991$ & $Q_{3}=0.36698$ \\
\hline
\end{tabular}

hole flux rate quite accurately. But for the calculation of the pressure in Peaceman well model, there are still some significant errors near the well, as shown in Fig. 6. For the proposed source term compensation method and the pattern competition method, both the bottom-hole flux rate and the pressure can be calculated accurately.

\section{Discussion}

In this paper, we figured out that for the widely used Peaceman well model, there are significant errors of pressure near the well for the large value of the length-to-width ratio of the mesh. Two methods, the source term compensation method and the pattern competition method are proposed for the homogeneous medium that can take place of the Peaceman well model. Both the two methods can calculate bottomhole flux rate and the pressure accurately for all the different grids. It is also found out that the simulation results of the source term compensation method are more accurate than the pattern competition method (see Fig. 6). However, the source term compensation method is difficult to be extended to heterogeneous medium, since the analytic Green function in heterogeneous medium is not available at present. On the other hand, the pattern competition method is more flexible and easier to be extended to heterogeneous cases. It is also suitable even for unstructured grids. Relevant issues for the extension of the proposed method to complex wells and to heterogeneous media are in the scope of future study.

\section{Acknowledgments}

This work is supported by National Science and Technology Major Project of China (No. 2017ZX05072-005) and the CNPC-CAS Science and Technology Cooperation Project (No. 2015A-4812).

Open Access This article is distributed under the terms and conditions of the Creative Commons Attribution (CC BY-NC-ND) license, which permits unrestricted use, distribution, and reproduction in any medium, provided the original work is properly cited.

\section{References}

Abou-Kassem, J.H., Aziz, K. Analytical well models for reservoir simulation. SPE J. 1985, 25(4): 573-579.

Artus, V., Fructus, D. Transmissibility corrections and grid control for shale gas numerical production forecasts. Oil Gas Sci. Technol. 2012, 67(5): 805-821. 
Chen, Z.X., Zhang, Y.Q. Well flow models for various numerical methods. Int. J. Numer. Anal. Model. 2009, 6(3): 375-388.

Correia, M.G., Maschio, C., Schiozer, D.J. Flow simulation using local grid refinements to model laminated reservoirs. Oil Gas Sci. Technol. 2018, 73(5).

Ding, D.Y. Near-well upscaling for reservoir simulations. Oil Gas Sci. Technol. 2004, 59(2): 157-165.

Ding, Y. A generalized 3D well model for reservoir simulation. SPE J. 1996, 1(4): 437-450.

Ding, Y., Jeannin, L. A multi-point flux approximation scheme for well modelling in reservoir simulations. ECMOR VII-7th European Conference on the Mathematics of Oil Recovery, Baveno, Italy, 5-8 September, 2000.

Ding, Y., Renard, G., Weill, L. Representation of wells in numerical reservoir simulation. SPE Reserv. Eval. Eng. 1998, 1(1): 18-23.

Dumkwu, F.A., Islam, A.W., Carlson, E.S. Review of well models and assessment of their impacts on numerical reservoir simulation performance. J. Pet. Sci. Eng. 2012, 82: 174-186.

Durlofsky, L.J. An approximate model for well productivity in heterogeneous porous media. Math. Geol. 2000, 32(4): 421-438.

Fumagalli, A., Scotti, A. An Efficient XFEM approximation of Darcy flows in arbitrarily fractured porous media. Oil Gas Sci. Technol. 2014, 69(4): 555-564.

Liu, Z.F., Wang, X.H. Finite analytic numerical method for two-dimensional fluid flow in heterogeneous porous media. J. Comput. Phys. 2013, 235: 286-301.

Mirzayev, M., Jensen, J.L. Interwell connectivity analysis in a low-permeability formation using a modified Capacitance Model with application to the East Pembina Field, Cardium Formation, Alberta. Oil Gas Sci. Technol. 2019, 74(26).

Mochizuki, S. Well productivity for arbitrarily inclined well. Paper SPE29133 Presented at the 13th Reservoir Simulation Symposium, San Antonio, Texas, 12-15 February, 1995.

Noetinger, B. About the determination of quasi steady state storativity and connectivity matrix of wells in 3D heterogeneous formations. Math. Geosci. 2016, 48(6): 641-662.

Noetinger, B., Zargar, G. Multiscale description and upscaling of fluid flow in subsurface reservoirs. Oil Gas Sci. Technol. 2004, 59(2): 119-139.

Palagi, C.L., Aziz, K. Modeling vertical and horizontal wells with Voronoi grid. SPE J. 1994, 9(1): 15-21.

Peaceman, D.W. Interpretation of well-block pressures in numerical reservoir simulation. SPE J. 1978, 18(3): 183194.

Peaceman, D.W. Interpretation of well-block pressures in numerical reservoir simulation with nonsquare grid blocks and anisotropic permeability. SPE J. 1983, 23(3): 531-543.

Peaceman, D.W. Representation of a horizontal well in numerical reservoir simulation. SPE Adv. Technol. Ser. 1993, 1(1): 7-16.

Peaceman, D.W. Interpretation of well-block pressures in numerical reservoir simulation: Part 3-off-center and multiple wells within a well block. SPE Reserv. Eng. 1990, 5(2): 227-232.

Peaceman, D.W. A new method for representing multiple wells with arbitrary rates in numerical reservoir simulation. SPE Reserv. Eng. 1995, 10(4): 253-258.

Peaceman, D.W. A new method for calculating well indexes for multiple wellblocks with arbitrary rates in numerical reservoir simulation. Paper SPE-79687-MS Presented at SPE Reservoir Simulation Symposium, Houston, Texas, 3-5 February, 2003.

Ribeiro, G.G., Maliska, C.R. Extension of Peaceman's and Ding's well indexes for application in 3D reservoir simulation with horizontal wells. Proceedings of the ENCIT 2014, 15th Brazilian Congress of Thermal Sciences and Engineering, Belém, Pará, Brazi, 10-13 November, 2014.

Weber, D.B., Hales, H.B., Baxter, L.L. A new method of formulating finite difference equations-some reservoir simulation examples. Paper PETSOC-2004-170 Presented at Canadian International Petroleum Conference, Calgary, Alberta, 8-10 June, 2004.

Williamson, A.S., Chappelear, J.E. Representing wells in numerical reservoir simulation: Part 1-theory. SPE J. 1981, 21(3): 323-338.

Wolfsteiner, C., Durlofsky, L.J., Aziz, K. Calculation of well index for nonconventional wells on arbitrary grids. Comput. Geosci. 2003, 7(1): 61-82. 